This item was submitted to Loughborough's Institutional Repository (https://dspace.lboro.ac.uk/) by the author and is made available under the following Creative Commons Licence conditions.

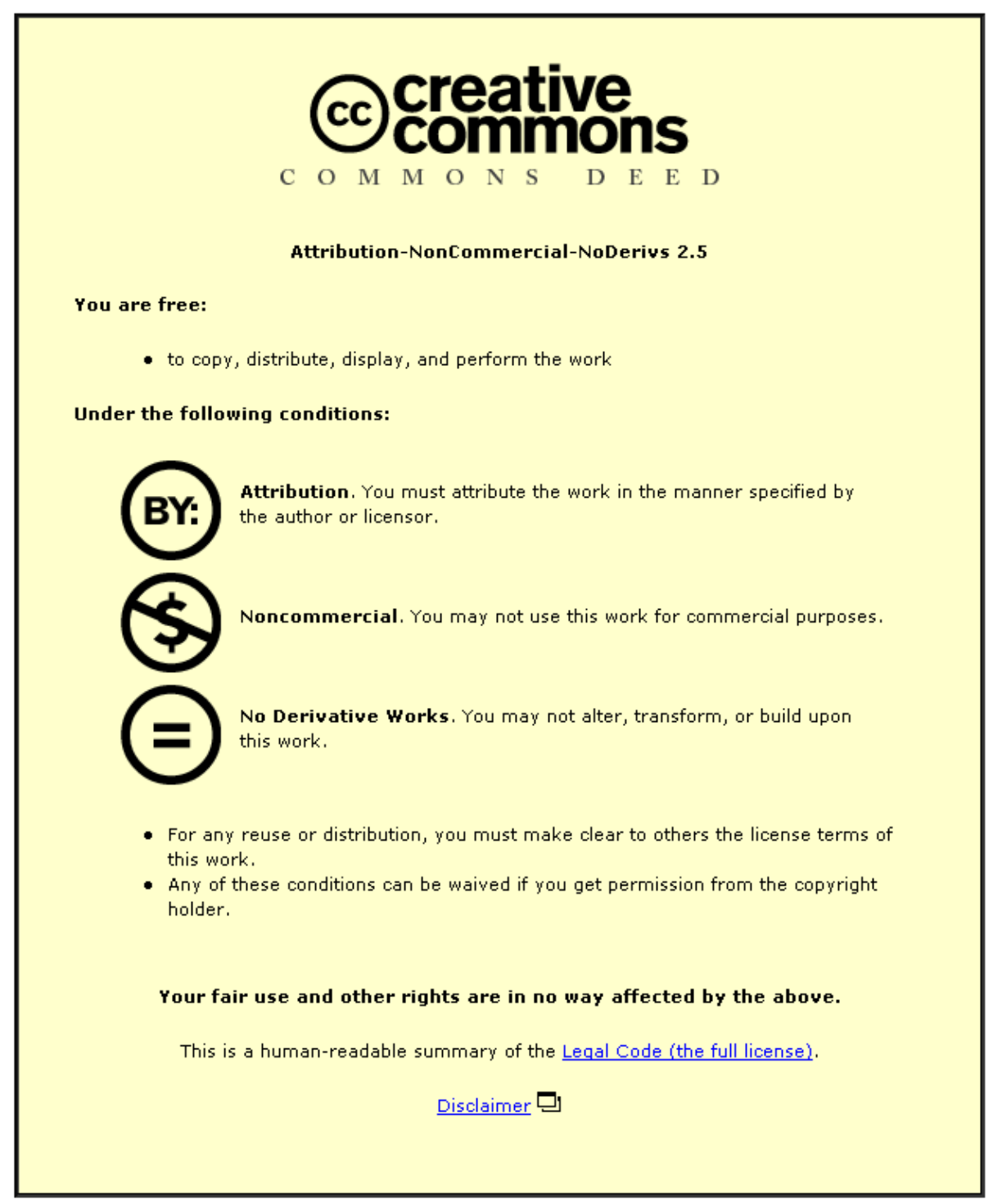

For the full text of this licence, please go to: http://creativecommons.org/licenses/by-nc-nd/2.5/ 


\title{
CONTAMINATION OF INDOOR AIR BY TOXIC SOIL VAPOURS: A SCREENING RISK ASSESSMENT MODEL
}

\author{
C.C FERGUSON*, V.V. KRYLOV* and P.T. McGRATH ${ }^{+}$ \\ Centre for Research into the Built Environment $\left(^{*}\right)$ and \\ Department of Building \& Environmental Health (+), \\ The Nottingham Trent University, \\ Burton Street, Nottingham NG1 4BU
}

\begin{abstract}
A simple steady-state model is derived for estimating the concentration of vapourphase contaminants in indoor air, given the contaminant concentration in the soil. The model includes the key mechanisms of transport and dispersion - contaminant partitioning into the soil-vapour phase, molecular diffusion, suction flow, and ventilation rate. It is shown that indoor air concentrations are largely controlled by two variables: soil permeability, and the half-life for contaminant removal from soil. A worked example shows the effect of these two variables on the concentration of benzene in soil that would give rise to a concentration of benzene in indoor air of 5 ppb (the recently proposed UK Air Quality Standard).
\end{abstract}

\section{INTRODUCTION}

The relatively high vapour pressures of many organic contaminants found in soils means that partitioning into the soil gas phase, and subsequent migration to human targets, needs to be 
considered in risk assessments of contaminated sites. Mixing with outdoor air results in large dilutions of gaseous contaminants so that their concentrations in the outdoor breathing zone are usually negligibly small. However, soil vapours migrating into living spaces of houses may reach concentrations that could be harmful to human health. Indeed, for some volatile organic compounds this may be the dominant pathway from source (contaminated soil) to target (the occupants of a house).

Modelling soil-vapour ingress into houses is not straightforward. For example, the model developed by Nazaroff et al [1] in the context of radon transport is specific to a particular style of North American house construction with basement sump and perimeter drain-tile system, and requires site-specific measurement for calibration. Our aim in this paper is to develop a very simple model that can be used more widely to give generic guidance on likely indoor vapour concentration for a given concentration of an organic contaminant in soil. The model can also take account of air contaminant contributions from other sources (e.g. stack emissions and road traffic, smoking in the living space and volatilisation of certain domestic chemicals inside the house). The value of such a model is that, combined with an appropriate safety or uncertainty factor, it can be used in screening assessments of contaminated sites. That is, it can help to identify sites where this pathway might be significant and hence where more detailed assessment would be warranted.

The model describes the equilibrium concentration of organic vapour assuming the construction style of a typical new-estate detached house in the U.K. (Fig. 1). The key mechanisms of transport and dispersion - contaminant partitioning into the soil-vapour phase, molecular diffusion, suction flow, and ventilation - are discussed in the following sections. 


\section{PARTITIONING INTO THE SOIL-VAPOUR PHASE}

Partitioning of organic contaminants between the solid, liquid and gaseous phases of soil is controlled by vapour pressure and aqueous solubility of the contaminant (or their ratio, which is Henry's Law constant) and by the partition coefficient between soil organic carbon and water, $K_{O C}$. The equilibrium contaminant concentration in the vapour phase, $C_{V}\left[\mu \mathrm{g} / \mathrm{cm}^{3}\right]$, is given by the simple equation [2]

$$
C_{V}=K_{b} C_{b}
$$

where

$$
K_{b}=\left[\left(K_{O C} \cdot f_{o c}\right) / H^{\prime}+S_{W} / \gamma H^{\prime}+S_{a} / \gamma\right]^{-1}
$$

Here $C_{b}[\mu \mathrm{g} / \mathrm{g}]$ is the contaminant concentration in bulk soil (reported according to the standard dry weight convention), $H^{\prime}$ is the dimensionless Henry's constant (see Jury et al [3] for values for many organic contaminants), $f_{O C}$ is the organic carbon fraction in soil, $\gamma$ is the specific gravity of the bulk (dry) soil, and $S_{w}$ and $S_{a}$ are, respectively, the water-filled and air-filled porosities of the soil.

\section{MOLECULAR DIFFUSION}

In the problem being considered diffusive fluxes are described using the simple linear relations

$$
q_{i j}=D_{i j}\left(C_{i}-C_{j}\right)
$$


where $q_{i j}$ is the flux $\left[\mathrm{g} / \mathrm{m}^{2} \mathrm{~h}\right]$ from compartment $\mathrm{i}$ to compartment $\mathrm{j}$. In the model (Figure 1) $\mathrm{i}$ and $\mathrm{j}$ take values 1, 2 or 3 denoting the following compartments:

$1:$ soil

2 : living space

3 : outdoor space

Contaminant vapour concentration in the relevant compartment is denoted $C_{i}$ or $C_{j}\left[\mathrm{~g} / \mathrm{m}^{3}\right]$, and the $D_{i j}$ are the coefficients of molecular diffusion $[\mathrm{m} / \mathrm{h}]$ for the material layers (floor, wall or ceiling) separating the relevant compartments. In what follows we will set the concentration of contaminant in the soil-vapour, $C_{1}$, equal to $C_{V}$ as calculated in equation (1). We now consider the diffusion coefficients in more detail.

\section{Diffusion through the floor}

We assume a floor construction (Figure 2) comprising a hard-core $(h c)$ base with sand(s) blinding, over which is placed a concrete (c) layer, an insulation layer (il), a PVC damp-proof sheet $(d p)$ and wooden decking $(w)$. The diffusion coefficient controlling diffusive flux from soil to living space, $D_{12}$, is thus calculated from the component diffusion coefficients identified by the above subscripts,

$$
1 / D_{12}=1 / D_{h c}+1 / D_{S}+1 / D_{C}+1 / D_{i l}+1 / D_{d p}+1 / D_{w}
$$


Each component coefficient is calculated as the effective molecular diffusivity $D_{\text {eff }}$ of the contaminant in the relevant material $\left[m^{2} / \mathrm{h}\right]$, divided by the layer thickness $d_{l}[\mathrm{~m}]$. We consider wooden decking to be perfectly penetrable $\left(1 / D_{w}=0\right)$. A very carefully layed and sealed PVC damp-proof layer is probably a rather efficient barrier for most organic vapours. In practice, its effective diffusivity is controlled by gaps, tears and puncture holes. We take the view that, for assessing long term exposure to contaminant vapours, it would be prudent to treat this layer as very leaky $\left(1 / D_{d p} \approx 0\right)$.

For the other materials, effective molecular diffusivity $D_{\text {eff }}$ is controlled by porosity of the medium. Thibodeaux \& Scott [4] give the following equation:

$$
D_{e f f}=D_{a} \frac{S_{a}^{10 / 3}}{S_{t}{ }^{2}}
$$

where $D_{a}$ is the molecular diffusivity in air, $S_{a}$ is the air-filled porosity and $S_{t}$ the total porosity (both in percent) of the medium. Calculations using (3) and (4) show that, for typical values of porosity and thickness, the coefficient $D_{12}$ has a value of about $5 \times 10^{-4} \mathrm{~m} / \mathrm{h}$ which is largely determined by the concrete layer.

\section{Diffusion through walls and ceiling}

We assume that house walls are constructed of a five-layer sandwich of brick $(b)$, air gap (ag), insulating layer (il), lightweight block (lb), and surface coating (sc). As before, the overall diffusion coefficient for the wall, $D_{23}(w)$, is determined by the components. Thus, neglecting the air gap where convection processes prevail, we have: 


$$
1 / D_{23(w)}=1 / D_{b}+1 / D_{i l}+1 / D_{l b}+1 / D_{S C} .
$$

Again, for typical values it is easy to show that the overall coefficient $D_{23}$ is largely determined by the brick layer. Coefficient of diffusion through the ceiling, $D_{23(c)}$, is calculated similarly but with a three layer sandwich - surface coating, plaster board, insulating layer:

$$
1 / D_{23(c)}=1 / D_{S C}+1 / D_{p b}+1 / D_{i l} .
$$

It is assumed that there is no effective diffusion barrier between the roof space and outside air, and hence the roof space is treated as part of the outside air (Figure 1).

\section{SUCTION FLOW}

Suction flow (sometimes called pressure-driven flow) results when the soil-gas pressure is greater than the air pressure inside a house. The pressure gradient causes chemical fluxes from soil to indoor air via connected pore spaces, gaps and cracks. Suction flow is likely to be important during the winter months but will probably be negligible in spring and summer when windows and doors are frequently open.

The velocity of air $V_{S}$ driven through a porous medium by a pressure gradient $\nabla P$ is determined from Darcy's law

$$
V_{S}=-\frac{k}{\mu} \nabla P,
$$


where $k$ is air permeability in the medium $\left[\mathrm{m}^{2}\right]$ and $\mu$ is the viscosity of air $\left[\mathrm{Nh} / \mathrm{m}^{2}\right]$. The corresponding flux of a chemical driven through the soil by this pressure gradient is

$$
q_{S}=V_{s} C_{1} / S_{a}
$$

The average value of the pressure gradient can be estimated using the simple formula

$$
\nabla P=\left(P_{2}-P_{1}\right) / d,
$$

where $P_{1}$ is the soil-gas pressure (taken as equal to the atmospheric pressure), $P_{2}$ is the air pressure inside the house, and $d$ is the average path length of the vapour molecules contributing to the contaminant flux between the compartments with pressures $P_{1}$ and $P_{2}$ (Figure 2). The characteristic path length is determined by depth of foundations, floor thickness, and location of high-diffusivity channels (gaps and cracks). We use $d=1 \mathrm{~m}$ as a default value. In winter a typical pressure difference is $P_{1}-P_{2}=3.5 P a \quad$ [1].

Air permeability ranges for different soils have been estimated by Johnson \& Etinger [5] as follows:

$$
\begin{array}{lrl}
\text { medium sand } & k & =10^{-11}-10^{-10} \mathrm{~m}^{2} \\
\text { fine sand } & k & =10^{-12}-10^{-11} \mathrm{~m}^{2} \\
\text { silty sand } & k & =10^{-13}-10^{-12} \mathrm{~m}^{2} \\
\text { silt } & k & =10^{-14}-10^{-13} \mathrm{~m}^{2}
\end{array}
$$

For clay soils we estimate an air permeability range of $10^{-16}-10^{-15} \mathrm{~m}^{2}$. 
Effective air permeability through the floor will be dominated by cracks and gaps. For simple generic modelling we assume that the floor will be so permeable relative to soil that suction flow through the soil-floor-wall system will be determined almost entirely by the soil.

\section{VENTILATION}

Natural ventilation is mainly caused by pressure differences between inside and outside air induced by aerodynamic flow (wind). Ventilation is critically important in determining vapour concentrations in indoor living spaces. The average ingoing and outgoing fluxes of a chemical vapour due to ventilation are simply

$$
\begin{aligned}
& q_{\text {vent }}{ }^{\text {in }}=V_{\text {vent }}{ }^{\text {in }} \cdot C_{3}, \\
& q_{\text {vent }}{ }^{\text {out }}=V_{\text {vent }}{ }^{\text {out. }} \cdot C_{2},
\end{aligned}
$$

where $V_{\text {vent }}$ in and $V_{\text {vent }}$ out are the velocities of ingoing and outgoing air averaged over all relevant gaps, holes, cracks and open doors and windows in the boundary of the living space. It is evident from mass conservation law that, at equilibrium, total ingoing and outgoing air fluxes are equal (note that total ingoing flux also includes a suction flow). This, however, does not relate to the total ingoing and outgoing fluxes of a chemical because of different values of concentrations $C_{2}$ and $C_{3}$. It is customary to express $V_{\text {vent }}$ in and $V_{\text {vent }}$ out in terms of air exchange rate:

$$
\begin{aligned}
& V_{\text {vent }}{ }^{\text {in }}=E_{X} \cdot V_{2} / A_{S}{ }^{\text {in }}-V_{s} A_{\text {suc }} / A_{s}^{\text {in }}, \\
& V_{\text {vent }}{ }^{\text {out }}=E_{X} \cdot V_{2} / A_{s} \text { out },
\end{aligned}
$$


Here $E_{X}$ is the number of air changes per hour in the total living space $V_{2}$, and $A_{S}$ in and $A_{S}$ out are the time-averaged surface areas of all relevant open holes, cracks, doors, windows etc at the boundary of $V_{2}$. Notation $A_{\text {suc }}$ describes the effective area of cracks and pores corresponding to the suction flow with the velocity $V_{s}$. Since $V_{s} A_{s u c} / V_{2}$ is negligibly small in comparison with $E_{X}$, in further derivations we will write $V_{v e n t}$ in simply as $E_{X} \cdot V_{2} / A_{S}$ in .

\section{INDOOR SOURCES OF CONTAMINATION}

In addition to contaminated soil and ambient outdoor air, other important sources of indoor air contamination may be located within the living space. For instance, cigarette smoking releases a range of toxic substances into the air either by direct smoking (mainstream smoke) or from smouldering cigarettes between puffs (sidestream smoke). Other sources of air contamination include domestic chemicals, such as paints, oils, washing liquids and powders, glues and cleaning fluids. In this paper, all indoor sources of air contamination are taken into account by means of their integral productivity per hour $[\mu \mathrm{g} / \mathrm{h}]$.

\section{EQUILIBRIUM CONCENTRATION OF TOXIC VAPOUR}

At equilibrium the air concentration of a chemical in each room of a house will be constant. Assuming that internal doors are opened sufficiently frequently to allow free interchange of air between rooms, one can consider the living space to be a single homogeneous volume $V_{2}$. In this case we derive the following balance equation (see Appendix): 


$$
\begin{aligned}
& q_{12} \cdot A_{f}+q_{S} \cdot P \cdot L+q_{v e n t}{ }^{\text {in }} \cdot A_{S}{ }^{i n}-q_{23}(w)^{\cdot}\left(A_{w a}-A_{w d}\right) \\
& -q_{23(c)} \cdot A_{C}-q_{v e n t} \text { out } A_{S} \text { out }+I=0
\end{aligned}
$$

Here $A_{f}$ and $A_{C}$ are the surface areas of floor and ceiling respectively (we assume $A_{f}=A_{C}$ ), $P$ is the perimeter length of the house, $L$ is a characteristic length over which suction flow is effective (so that the area through which suction flow takes place is $P L$ ), and the indices $(w)$ and (c) attached to 923 specify fluxes through walls and ceiling respectively. Similarly, the relevant areas are denoted by subscripts wa (walls), wd (windows and doors) $c$ (ceiling) and $s$ (time-averaged surface area of open holes etc). Note that in equation (12) windows and doors are considered as impenetrable by organic vapours. Any key-holes, gaps at the margins of doors and windows etc are taken into account through the ventilation fluxes $q_{v e n t}{ }^{\text {in }}$ and $q_{\text {vent }}$ out. The time-averaged production of air contaminants from indoor sources is denoted I.

Substituting equations (2) and (7)-(11) into equation (12) we can write

$$
\begin{gathered}
D_{12}\left(C_{1}-C_{2}\right) A_{f}+V_{s} C_{1} P \cdot L / S_{a}-D_{23(w)}\left(C_{2}-C_{3}\right)\left(A_{w a}-A_{w d}\right)- \\
D_{23(c)}\left(C_{2}-C_{3}\right) A_{C}-E_{X} A_{f} h \cdot\left(C_{2}-C_{3}\right)+I=0
\end{gathered}
$$

where $\mathrm{h}$ is the total height of the living space. Recalling that $C_{1}=C_{V}$ and expressing $C_{V}$ in terms of contaminant concentration in bulk soil $C_{b u l k}$ (see eqn (1)) we solve (13) to obtain the following expression for concentration of toxic vapour in the living space:

$$
C_{2}=\frac{\left(D_{12}+V_{s} P \cdot L / S_{a} A\right) K_{b} C_{b}+\left[D_{23(w)}\left(A_{w a}-A_{w d}\right) / A+D_{23(c)}+E_{X} h\right] C_{3}+I / A}{D_{12}+D_{23(w)}\left(A_{w a}-A_{w d}\right) / A+D_{23(c)}+E_{X} h} .
$$


Formula (14) describes the equilibrium indoor concentration of a contaminant as a function of contaminant concentration in the bulk soil and in outdoor air, as well as of total productivity of indoor sources of contamination.

In the limiting case of no indoor contaminant sources $(I=0)$, unpolluted outdoor air $\left(C_{3}=0\right)$ and polluted soil $\left(C_{b^{\neq}}\right)$it follows from (14) that

$$
C_{2}=\frac{\left(D_{12}+V_{s} P \cdot L / S_{a} A\right) K_{b}}{D_{12}+D_{23(w)}\left(A_{w a}-A_{w d}\right) / A+D_{23(c)}+E_{X} h} C_{b}
$$

In another limiting case of unpolluted soil $\left(C_{b}=0\right)$ and polluted outdoor air $\left(C_{3} \neq 0\right)$ in the absence of indoor sources $(I=0)$, eqn (14) is reduced to

$$
C_{2}=\frac{\left[D_{23(w)}\left(A_{w a}-A_{w d}\right) / A+D_{23(c)}+E_{X} h\right]}{D_{12}+D_{23(w)}\left(A_{w a}-A_{w d}\right) / A+D_{23(c)}+E_{X} h} C_{3} .
$$

If the ventilation term $E_{X} h$ in (16) is much larger than all other terms both in the nominator and in the denominator (the usual case), then it follows from (16) that $C_{2} \approx C_{3}$ as expected. In the general case both the polluted soil and outdoor air contributions are important, and eqn (14) must be used to calculate the indoor concentration.

To conclude this section, we consider three other limiting cases in the presence of indoor sources $(I \neq 0)$. For unpolluted outdoor air $\left(C_{3}=0\right)$ we have

$$
C_{2}=\frac{\left(D_{12}+V_{s} P \cdot L / S_{a} A\right) K_{b} C_{b}+I / A}{D_{12}+D_{23(w)}\left(A_{w a}-A_{w d}\right) / A+D_{23(c)}+E_{X} h} .
$$


and for unpolluted soil $\left(C_{b}=0\right)$,

$$
C_{2}=\frac{\left[D_{23(w)}\left(A_{w a}-A_{w d}\right) / A+D_{23(c)}+E_{X} h\right] C_{3}+I / A}{D_{12}+D_{23(w)}\left(A_{w a}-A_{w d}\right) / A+D_{23(c)}+E_{X} h} .
$$

For unpolluted outdoor air $\left(C_{3}=0\right)$ and unpolluted soil $\left(C_{b}=0\right)$ formula (14) takes the especially simple form

$$
C_{2}=\frac{I / A}{D_{12}+D_{23(w)}\left(A_{w a}-A_{w d}\right) / A+D_{23(c)}+E_{X} h},
$$

which can be used to estimate the influence of indoor sources alone.

\section{WORKED EXAMPLE: BENZENE VAPOUR IN INDOOR AIR}

We use equation (14) to calculate a first estimate of the expected concentration of benzene in the living space of a house for different values of its concentration in soil and in outdoor air,

and for different productivities of indoor sources of contamination. We are particularly concerned to identify the conditions under which the concentration of benzene vapour inside a typical new detached house might exceed a safety guideline such as the recently proposed UK Air Quality Standard of 5 ppb running annual average [6]. Data used in the calculation are summarised in Table 1.

Effective molecular diffusivities are calculated from equation (4) with the molecular diffusivity of benzene in air taken as $D_{a}=1.8 \times 10^{-2} \mathrm{~m}^{2} / \mathrm{h}$ [1]. Then, using the layer 
thickness values in Table 1, diffusion coefficients for the floor layers are calculated as follows:

$\begin{array}{llll}\text { hardcore: } & D_{h c}= & 7.0 \times 10^{-3} \mathrm{~m} / \mathrm{h} \\ \text { blinding sand: } & D_{S}= & 1.43 \times 10^{-1} \mathrm{~m} / \mathrm{h} \\ \text { concrete: } & D_{C}=4.96 \times 10^{-4} \mathrm{~m} / \mathrm{h} \\ \text { insulating layer: } & D_{i l}=3.13 \times 10^{-1} \mathrm{~m} / \mathrm{h} .\end{array}$

Substituting these values into equation (3) gives the following value for the coefficient of molecular diffusion $D_{12}$ between the soil and living space compartments:

$$
D_{12}=4.61 \times 10^{-4} \mathrm{~m} / \mathrm{h} \text {. }
$$

Thus $D_{12}$ is determined mainly by the concrete layer.

Similarly the coefficients of molecular diffusion between the living space and outdoor air compartments, $D_{23}$, through walls (w) and ceiling $(c)$ are:

$$
\begin{aligned}
& D_{23}(w)=3 \times 10^{-3} \mathrm{~m} / \mathrm{h} \\
& D_{23}(c)=2.1 \times 10^{-2} \mathrm{~m} / \mathrm{h} \text {. }
\end{aligned}
$$

Suction flow velocity $V_{S}$ is calculated from equations (7) - (9) using a typical pressure difference in winter of $P_{1}-P_{2}=3.5 P a$, a default value for the average path length of the flux beneath outside walls of $d=1 \mathrm{~m}$, and an air viscosity of $\mu=5 \times 10^{-9} \mathrm{Nh} / \mathrm{m}^{2}$. The characteristic length $L$ over which suction flow is effective is taken as $L=0.5 \mathrm{~m}$. Thus 
using a conservative choice for the value of permeability $\left(k=10^{-11} \mathrm{~m}^{2}\right.$ representing medium-sand soils) we get $V_{S}=7.0 \times 10^{-3} \mathrm{~m} / \mathrm{h}$.

To illustrate the relative importance of suction flow in comparison with diffusion flow we first estimate the benzene concentration in the living space, $C_{2}$, in terms of bulk soil concentration $C_{b}$ in the absence of a suction flow $\left(V_{S}=0\right.$, typical for summer time). We also assume that outdoor air is unpolluted $\left(C_{3}=0\right)$ and there is no indoor sources of benzene vapour $(I=0)$. Let us also consider that the living space is ventilated by one air exchange per hour: $E_{X}=1 h^{-1}[11]$. Then from equations (14) or (15) we calculate $C_{2}=2.1 \times 10^{-5} C_{b}$. In winter time, when suction flow is present, the analogous calculation is $C_{2}=3.91 \times 10^{-4} C_{b}$. Thus we expect benzene vapour concentration to be about 19 times smaller in summer than in winter because of the influence of a suction flow.

We now calculate the benzene concentration in bulk soil that would give a running annual average of $C_{2}=5 \mathrm{ppb}$ benzene in the living space when outdoor air is unpolluted $\left(C_{3}=0\right)$ and indoor sources are absent $(I=0)$. We first convert from $p p b$ to $\mu \mathrm{g} / \mathrm{cm}^{3}$ by multiplying by the mass density of benzene vapour $\left(3240 \mu \mathrm{g} / \mathrm{cm}^{3}\right)$ : thus the Air Quality Standard for benzene can be written as $C_{2 m}=1.62 \times 10^{-5} \mu \mathrm{g} / \mathrm{cm}^{3}$. Assuming for simplicity that summer and winter each last 6 months, the running annual average of the indoor concentration $<C_{2}>$ may be written in the form

$$
<C_{2}>=\left[\left(K_{w}+K_{S}\right) / 2\right] C_{b},
$$

where 


$$
K_{w}=\frac{\left(D_{12}+V_{s} P \cdot L / S_{a} A\right) K_{b}}{D_{12}+D_{23(w)}\left(A_{w a}-A_{w d}\right) / A+D_{23(c)}+E_{X} h}
$$

is a winter time proportionality coefficient, and

$$
K_{S}=\frac{D_{12} K_{b}}{D_{12}+D_{23(w)}\left(A_{w a}-A_{w d}\right) / A+D_{23(c)}+E_{X} h}
$$

is a summer time proportionality coefficient. Replacing $\left\langle C_{2}>\right.$ in (20) by $C_{2} m$ and solving gives $C_{b}=C_{b m}=0.079 \mu \mathrm{g} / \mathrm{g}$.

This calculation, however, ignores the gradual reduction of benzene concentration in soil due to volatilisation and other decay processes such as biodegradation. It is vital to take such processes into account if the objective is to estimate the maximum permissible concentration of benzene in soil now that will not exceed a prescribed average level of exposure via indoor air averaged over a lifetime. We assume that benzene reduction is a first-order decay process,

$$
d C_{b} / d t=-\alpha C_{b}
$$

which can be solved to give

$$
C_{b}(t)=C_{b}(0) \exp (-\alpha t) .
$$

Hence the average concentration in soil over the time interval $\Delta t=t_{\text {max }}-t_{O}$ is given by

$$
<C_{b}>=\frac{C_{b}(0)}{\Delta t} \int_{t_{0}}^{t_{\max }} e^{-\alpha t} d t,
$$


and the initial concentration $C_{b}(0)$ that will give this average is

$$
C_{b}(0)=\frac{\alpha \Delta t<C_{b}>}{e^{-\alpha t_{0}}-e^{-\alpha t_{\max }}}
$$

Without loss of generality, in further calculations we will put $t_{0}=0$. The decay constant $\alpha$ is related to the half-life $t_{1 / 2}$ of a compound in soil by the equation $t_{1 / 2}=\ln (2) / \alpha$. Estimates of biological/chemical degradation half-lives for benzene range from 20 days [7] to 1 year [3]. However it is known that many organic compounds with nominally short half-lives do persist in soil. Laboratory measurements of single compound degradation kinetics in synthetic soils cannot be used to provide even rough estimates of natural degradation rates of the same compound occurring in mixtures. Also, simple calculations of rate of loss from soil ignore the fact that the metabolites of a chemical might also have adverse effects, and might even be more toxic than the precursor chemical. Possible metabolites of benzene (e.g. phenol, catechol, hydroquinine) are generally considered to be less toxic than benzene, although there is some evidence that the toxicity of benzene in humans is effected through these metabolites [8].

Given the many uncertainties in choosing an appropriate half-life for benzene, we believe that a suitably conservative choice is $t_{1 / 2}=1$ year. Thus, if we set the average concentration in soil, $\left\langle C_{b}>\right.$, at the level corresponding to an average benzene concentration of $5 \mathrm{ppb}$ in living space air (i.e. $\left\langle C_{b}>=0.079 \mu \mathrm{g} / \mathrm{g}\right.$ ) we obtain the following initial benzene concentration in soil,

$$
C_{b}(0)=3.81 \mu \mathrm{g} / \mathrm{g}
$$


This result can be considered as providing an upper bound estimate for a permissible concentration of benzene in soil because the calculation neglects exposure pathways other than inhalation of indoor air (although these are probably relatively minor).

Since the value $C_{b}(0)$ is very sensitive to soil permeability $\mathrm{k}$ and half-life of a chemical $t_{1 / 2}$ it is convenient to represent $C_{b}(0)$ for the example considered as a function of only these two parameters. Then it follows from (7)-(9), (15), (20) and (24) that $C_{b}(0)$ may be written in the following simplified form:

$$
C_{b}(0)=\frac{2 \ln (2) \Delta t C_{2 m}}{t_{1 / 2}\left[1-\exp \left(-\ln (2) \Delta t / t_{1 / 2}\right)\right]\left(4.210^{-5}+3.710^{7} \mathrm{k}\right)},
$$

where $k$ is in $m^{2}$ and $t_{1 / 2}$ in years. Concentration $C_{b}(0)$ as a function of soil permeability, $v=\log (k)$, is shown on Fig. 3 for different values of a half-life $t_{1 / 2}$. One can see that in the range of $v$ from -12.5 to -11 the value of $C_{b}(0)$ is very sensitive to $v$, especially for lower half-lifes. Figure 4 shows the behaviour of $C_{b}(0)$ as a function of halflife for different values of soil permeability. It is seen that the highest sensitivities to $t_{1 / 2}$ occur at the smallest $t_{1 / 2}$ and lowest permeabilities. For $k=10^{-11} \mathrm{~m}^{2}$ and $t_{1 / 2}=1$ year both figures give the result (25), as expected.

Let us now discuss the additional influence of benzene-contaminated ambient air $\left(C_{3} \neq 0\right)$ entering a house due to normal ventilation processes (indoor sources of contamination are again assumed to be absent: $I=0$ ). For the purposes of demonstrating a simple lifetime exposure model we will assume a constant concentration of benzene in ambient air, and a contribution from soil reflecting exponential decay of benzene in soil with time. It is 
convenient to display the results in normalised form (Figure 5) in which ordinate values are multiples of the proposed Air Quality Standard (that is multiples of $5 p p b$ ). Abscissa values are fractions of the initial soil concentration $C_{b}(0)$ that would give an indoor air concentration of $5 \mathrm{ppb}$ if ambient air was unpolluted; progressive soil clean-up from that level would be represented by moving from right to left on the plot. The additive nature of these two components is apparent from the plot, which reinforces the obvious conclusion that clean-up can have a significant influence on total benzene concentration when the ambient concentration is low (say $1-2 p p b$ ). But when ambient concentrations are higher, the proportional gain from soil clean-up might be more difficult to justify on a comparative costbenefit basis.

We now consider the influence of indoor sources of contamination $(I \neq 0)$ such as smoking cigarettes and volatilisation of domestic chemicals, in the absence of benzene-contaminated ambient air $\left(C_{3}=0\right)$. The average amount of benzene released into the atmosphere via mainstream and sidestream cigarette smoke may be taken as $300 \mu \mathrm{g}$ per cigarette [9]. If it is assumed that 20 cigarettes are smoked inside a home each day, the total benzene productivity would be $I_{s}=(300 \mu g \times 20) / 24 h=250 \mu g / h$. We assume, arbitrarily, that other sources of benzene such as household chemicals will contribute an additional $50 \mu \mathrm{g} / \mathrm{h}$ making a total of $I=300 \mu \mathrm{g} / \mathrm{h}$. As before, we consider a contribution from soil reflecting exponential decay of benzene in soil with time. The results are displayed in normalised form (Figure 6) in which ordinate values are multiples of the proposed Air Quality Standard as in Figure 5. Abscissa values are fractions of the initial soil concentration $C_{b}(0)$ that would give an indoor air concentration of $5 p p b$ if indoor sources were absent. As in Figure 5, progressive soil clean-up from that level would be represented by moving from right to left on the plot. 
One can see that for $I=300 \mu \mathrm{g} / \mathrm{h}$ the indoor sources contribution to indoor benzene concentration is small compared with the soil contribution. Even a value ten times as large (I $=3000 \mu \mathrm{g} / \mathrm{h}$ ) would only contribute about one-half of the Air Quality Standard in the absence of benzene from soil vapour.

The above conclusion about the small influence of indoor sources on equilibrium indoor concentration of benzene is valid for a typical air exchange rate of $E_{x}=1 h^{-1}$. For smaller $E_{x}$ indoor sources will be more important. Figure 7 shows the indoor concentration of benzene (in $\mathrm{ppb}$ ) as a function of $E_{X}$ for two values of indoor sources productivity: $I=300$ and $600 \mu \mathrm{g} / \mathrm{h}$ (concentration of benzene in outdoor air was chosen as $C_{3}=2 \mathrm{ppb}$ ). One can see that for low values of $E_{X}$ the influence of indoor sources is very large. However, for $E_{X}$ $>0.3$ their influence is small and the resulting indoor concentration, as expected, approaches that of ambient outdoor air.

\section{CONCLUSIONS}

Penetration of toxic soil vapours into the living spaces of houses can lead to potential health risks under some conditions. The simple steady-state model developed in this paper confirms that the most critical variables for long term risk assessment are soil permeability and the half-life for removal of the chemical from soil. Uncertainties about appropriate values for these two variables are likely to dominate any application of this model for risk assessment. A worked example with benzene is used to illustrate the importance of these variables. 
Figure 3 shows that, for a benzene half-life choice of 1 year, the soil concentration leading to $5 p p b$ of benzene in indoor air (assuming unpolluted ambient air) is very strongly dependent on soil type (soil permeability). But for any reasonable choice of half-life the acceptable level for sandy soils (say $k \geq 10^{-10.5} \mathrm{~m}^{2}$ ) is around $1 \mu \mathrm{g} / \mathrm{g}$ or less. Given the uncertainties in any generic model of this kind, we believe that for sandy soils a benzene concentration greater than $0.2 \mu \mathrm{g} / \mathrm{g}$ in soil ought to trigger further (and site-specific) consideration. For silty soils $\left(k \leq 10^{-13} \mathrm{~m}^{2}\right)$ benzene concentrations in soil which might give $5 \mathrm{ppb}$ in indoor air range from about $7 \mu \mathrm{g} / \mathrm{g}$ (for a half-life of 5 years) to more than $30 \mu \mathrm{g} / \mathrm{g}$ (for a half-life of 1 year). If we assume (arbitrarily) that no more than one-quarter of the Air Quality Standard should be contributed by soil, then maximum acceptable concentrations for silty soils would be roughly in the range $2-8 \mu \mathrm{g} / \mathrm{g}$. Prudence would suggest using the lower value. Clay soils might have such low natural permeabilities that lifetime vapour intake from this source would be expected to be negligible. However, field transmissivity - taking into account fractures and macropores - may be much greater than that predicted from permeability measured under laboratory conditions. Thus it may be prudent to recommend the same generic guideline for clay soils as for silty soils.

Measurements of benzene concentration in 100 houses over a 4 week exposure period [10] suggest that the proposed Air Quality Standard (AQS) of $5 \mathrm{ppb}$ is likely to be exceeded in many UK homes. The UK Expert Panel on Air Quality Standards has recommended that the long-term aim should be to reduce the benzene AQS from $5 p p b$ to $1 p p b$ [6]. This is likely to raise difficult questions about appropriate remedial strategies. In this paper we have discussed non-soil sources of benzene (outside ambient air, indoor sources such as cigarette smoking) to show their effect on total indoor benzene concentration in the event of remedial action to 
reduce benzene concentration in soil. This reinforces the obvious point - that when non-soil sources dominate, it makes either logical or economic sense to tackle the problem of excess benzene in indoor air by imposing stringent soil clean-up requirements.

\section{ACKNOWLEDGEMENTS}

This work forms part of a research project funded by the UK Department of the Environment. We are grateful for critical comments from the Department of the Environment's Toxic Substances Division, the Environmental Physics Division of the Building Research Establishment, Dr. A. Aroussi (University of Nottingham), and the journal's anonymous referees. However, the opinions expressed in this paper are those of the authors alone and should not be taken to represent those of the Department of the Environment.

\section{REFERENCES}

1. W. W. Nazaroff, H. Feustel, A. V. Nero, K. L. Rezvan, D. T. Grimsrud, M. A. Essling and R. E. Toohey, Radon transport into a detached one-story house with a basement, Atmosph. Env., 19, 31-46 (1985).

2. W. A. Jury, W. F. Spencer and W. J. Farmer, Behaviour assessment model for trace organics in soil: I, Model description, J. Env. Quality, 12, 558 - 564 (1983). 
3. W. A. Jury, D. Russo, G. Streile and E. A. Hesham, Evaluation of votalization by organic chemicals residing below the soil surface, Water Resource Research, 26, 13 20 (1990).

4. L. J. Thibodeaux and H. D. Scott, Air/soil exchange coefficients, In: Environmental Exposure from Chemicals, W.B. Neely and G.E. Blau, eds. Vol.1, CRC Press, Boca Raton (USA), (1985).

5. P. C. Johnson and R. A. Etinger, Heuristic model for predicting the intrusion rate of contaminated vapour into buildings, Env. Sci. Technol., 25, 1445-1452 (1991).

6. Department of the Environment, Expert Panel on Air Quality Standards: Benzene, H.M.S.O., London (1994).

7. D. J. Paustenbach, R. Kalmes, J. D. Jernigan, R. Bass and P. Scott, A proposed approach to regulating contaminated soil: identify safe concentrations for seven of the most frequently encountered exposure scenarios, In: Calabrese, E.J. and Kosteckis, P.T. Principles and Practises for Petroleum Contaminated Soils, Lewis Publishers, Chelsea, MI, pp 511 - 552. (1993).

8. R. Snyder, E.A. Dimitriadis, R. Guy, P. Hu, K. Cooper, H. Bauer, G. Witz and B.D. Goldstein, Studies on the mechanism of benzene toxicity, Environ Health Perspect., 82, 31 - 35 (1989).

9. National Research Council, Committee on Passive Smoking, Board on Environmental Studies and Toxicology, Environmental tobacco smoke: measuring exposures and assessing health effects, National Academic Press (1986).

10. V. M. Brown, D. R. Crump, D. Gardiner and C. F. F. Yu, Long term diffusive sampling of volatile organic compounds in indoor air, Environmental Technology,14, 771-777 (1993). 
11. E. Perera, and L. Parkins, Airtightness of UK buildings: Status and future possibilities, In: Environmental Policy and Practice, BRE, 2, No 2, 143-160 (1992).

\section{APPENDIX \\ DERIVATION OF THE GOVERNING EQUATION}

In the presence of indoor sources of a polluting gas in a macroscopic point considered, the differential equation describing time and space evolution of gas concentration may be written in the form

$$
\partial C / \partial=\left(\nabla \cdot D_{e f f} \nabla C\right)-(\nabla \cdot C v) / S_{a}+J
$$

Here $C$ is concentration of a chemical in air, $D_{\text {eff }}$ is effective molecular diffusivity, $\mathbf{v}$ is the velocity vector of a hydrodynamic flow, $S_{a}$ is the air-filled porosity (where appropriate), and $J$ is the density of indoor sources of a polluting gas. Note that in the absence of hydrodynamic flows $(v=0)$ or gradient of concentration $(\nabla C=0)$ equation (A1) reduces respectively either to the equation of diffusion or to the hydrodynamic continuity equation for porous media written in terms of concentration. 
Integrating eqn (A1) over the volume $V$ bounded by the closed surface $S$ (surface $S$ may be chosen, e.g., inside the outer material boundaries of the house (floor, walls and ceiling) and using divergention theorem, one can get

$$
(\partial / \partial) \int_{V} C d V=\oint_{S} D_{e f f}(\boldsymbol{n} \cdot \nabla C) d S-\oint_{S} C(\boldsymbol{v} \cdot \boldsymbol{n}) d S+\int_{V} J d V
$$

where $\boldsymbol{n}$ is a vector of unit normal to the surface directed outside the closed volume. For steady-state or very slowly-varying processes we are interested in, the term with time derivative in (A2) can be neglected, and it follows from eqn (A2) that

$$
\oint_{S} D_{e f f}(\boldsymbol{n} \cdot \nabla C) d S-\oint_{S} C(\boldsymbol{v} \cdot \boldsymbol{n}) d S+I=0
$$

where we have introduced the notation $I=\int_{V} J d V$ for the total productivity of indoor sources of a polluting gas. Note that quantities $D_{\text {eff }}, \nabla C$ and $v$ in eqn (A3) are generally functions of the position on the surface.

In further transformations we use standard definitions of diffusive and hydrodynamic fluxes:

$$
\begin{aligned}
& \boldsymbol{q}^{\text {dif }}=-D_{e f f} \nabla C \\
& \boldsymbol{q}^{h y d}=C v / S_{a}
\end{aligned}
$$

and will transfer from the mathematical surface $S$ to the corresponding "material surface" having a finite thickness and reflecting the physical properties of walls, floor and ceiling of a house. The absolute values of diffusive fluxes crossing the material surface may be written in 
the form $q$ dif $=D\left(C_{\text {out }}-C_{i n}\right)$, where $D$ is a position-dependent diffusion coefficient for the material boundary. Then we split general position-dependent diffusive flux $q$ dif on ingoing and outgoing diffusion fluxes coming through the floor from the polluted ground (index 1) to the inner volume of the house (index 2), $q_{12}$, and through the walls (index "w") and ceiling (index "c") from the volume of the house to the outer space (index 3), $q_{23(w)}$ and $q_{23}(c)$. In a similar way, we specify the general position-dependent hydrodynamic flux $\boldsymbol{q}^{\text {hyd }}$ as an ingoing suction flux from the ground into the house, $q_{S}$, and as ingoing and outgoing ventilation fluxes, $q_{v e n t}{ }^{\text {in }}$ and $q_{v e n t}{ }^{\text {out }}$. Using these specifications in eqns (A3), (A4) and replacing integration over $S$ by multiplication over relevant surface areas corresponding to the above specified spatially homogeneous parts of the general diffusive and hydrodynamic fluxes, one can easily obtain equation (12) of the main text which is used for calculating the concentration $C_{2}$ of toxic vapor inside a house. 
TABLE 1

\section{Data used in worked example}

\begin{tabular}{|c|c|}
\hline \multicolumn{2}{|l|}{ Soil parameters } \\
\hline Specific gravity of dry soil: & $\gamma=1.6$ \\
\hline Air-filled porosity: & $S_{a}=0.2$ \\
\hline Water-filled porosity: & $S_{W}=0.1$ \\
\hline Organic carbon fraction: & $f_{O C}=0.01$ \\
\hline \multicolumn{2}{|c|}{ Benzene parameters (Jury et al 1990) } \\
\hline \multicolumn{2}{|c|}{$\begin{array}{ll}\text { Partition coefficient between soil organic carbon and water: } & K_{O C}=80 \mathrm{~cm}_{3} / \mathrm{g} \\
\text { Dimensionless Henry's constant: } & H^{1}=0.22\end{array}$} \\
\hline \multicolumn{2}{|c|}{ House parameters } \\
\hline Volume of living space: & $V_{2}=400 \mathrm{~m}^{3}$ \\
\hline Height of living space: & $h=5.4 m$ \\
\hline Perimeter of house: & $p=34.4 m$ \\
\hline Surface area of internal windows and doors: & $A_{w d}=20 m_{2}$ \\
\hline Air exchange rate in the living space: & $E_{X}=1 h^{-1}$ \\
\hline Thickness of hard-core layer & $0.1 \mathrm{~m}$ \\
\hline Thickness of blinding sand & $0.05 \mathrm{~m}$ \\
\hline Thickness of concrete floor layer & $0.1 \mathrm{~m}$ \\
\hline Thickness of floor insulating layer & $0.05 \mathrm{~m}$ \\
\hline Thickness of brick layer in walls & $0.1 \mathrm{~m}$ \\
\hline Thickness of lightweight block layer in walls & $0.1 \mathrm{~m}$ \\
\hline Thickness of insulating layer in walls & $0.055 \mathrm{~m}$ \\
\hline Thickness of surface coating on walls & $0.001 \mathrm{~m}$ \\
\hline Thickness of ceiling plasterboard & $0.022 \mathrm{~m}$ \\
\hline Thickness of roof insulating layer & $0.1 \mathrm{~m}$ \\
\hline Thickness of ceiling surface coating & $0.001 \mathrm{~m}$ \\
\hline \multicolumn{2}{|l|}{ Building material properties } \\
\hline Air-filled porosity of hardcore & $25 \%$ \\
\hline Total porosity of hardcore & $50 \%$ \\
\hline Air-filled porosity of blinding sand & $50 \%$ \\
\hline Total porosity of blinding sand & $50 \%$ \\
\hline Air-filled porosity of concrete & $3.4 \%$ \\
\hline Total porosity of concrete & $6.8 \%$ \\
\hline Air-filled porosity of insulating layer & $90 \%$ \\
\hline Total porosity of insulating layer & $90 \%$ \\
\hline Air-filled porosity of brick & $25 \%$ \\
\hline Total porosity of brick & $50 \%$ \\
\hline Air-filled porosity of plasterboard & $6.8 \%$ \\
\hline Total porosity of plasterboard & $6.8 \%$ \\
\hline
\end{tabular}




\section{FIGURE CAPTIONS}

Fig.1. Schematic diagram of the house considered. Arrows indicate ingoing and outgoing fluxes of a chemical

Fig.2 Schematic diagram showing construction details of a floor and adjacent wall. Arrows show the path and direction of suction flow

Fig.3. Initial bulk-soil concentration of benzene, $C_{b}(0)$, as a function of the soil permeability index $v$ : $\left(k=10^{v} \mathrm{~m}^{2}\right)$ for different half-lives (years) as labelled on curves

Fig.4. Initial bulk-soil concentration of benzene, $C_{b}(0)$, as a function of removal half-life, $t_{1 / 2}$ (in years), for different values of soil permeability index $v$ as labelled on curves

Fig.5. Influence of outdoor air concentration of benzene (as labelled on curves) on the indoor air concentration expressed as a ratio of the proposed Air Quality Standard (i.e. a value of 1 is equivalent to $5 p p b$; a value of 2 is equivalent to $10 p p b$, etc). Abscissa values are scaled to the soil concentration that would give $5 p p b$ in indoor air in the absence of benzene in ambient air

Fig.6. Influence of indoor sources productivity (as labelled on curves) on the indoor air concentration of benzene expressed as a ratio of the proposed Air Quality Standard 
(i.e. a value of 1 is equivalent to $5 p p b$; a value of 2 is equivalent to $10 p p b$, etc). Abscissa values are scaled to the soil concentration that would give $5 p p b$ in indoor air in the absence of benzene in ambient air

Fig.7. Indoor air concentration of benzene (in $p p b$ ) as a function of air exchange rate due to ventilation, $E_{X}$, for two values of indoor sources productivity (as labelled on curves); outdoor air concentration of benzene, $C_{3}$, is chosen as $2 p p b$ 
Figures

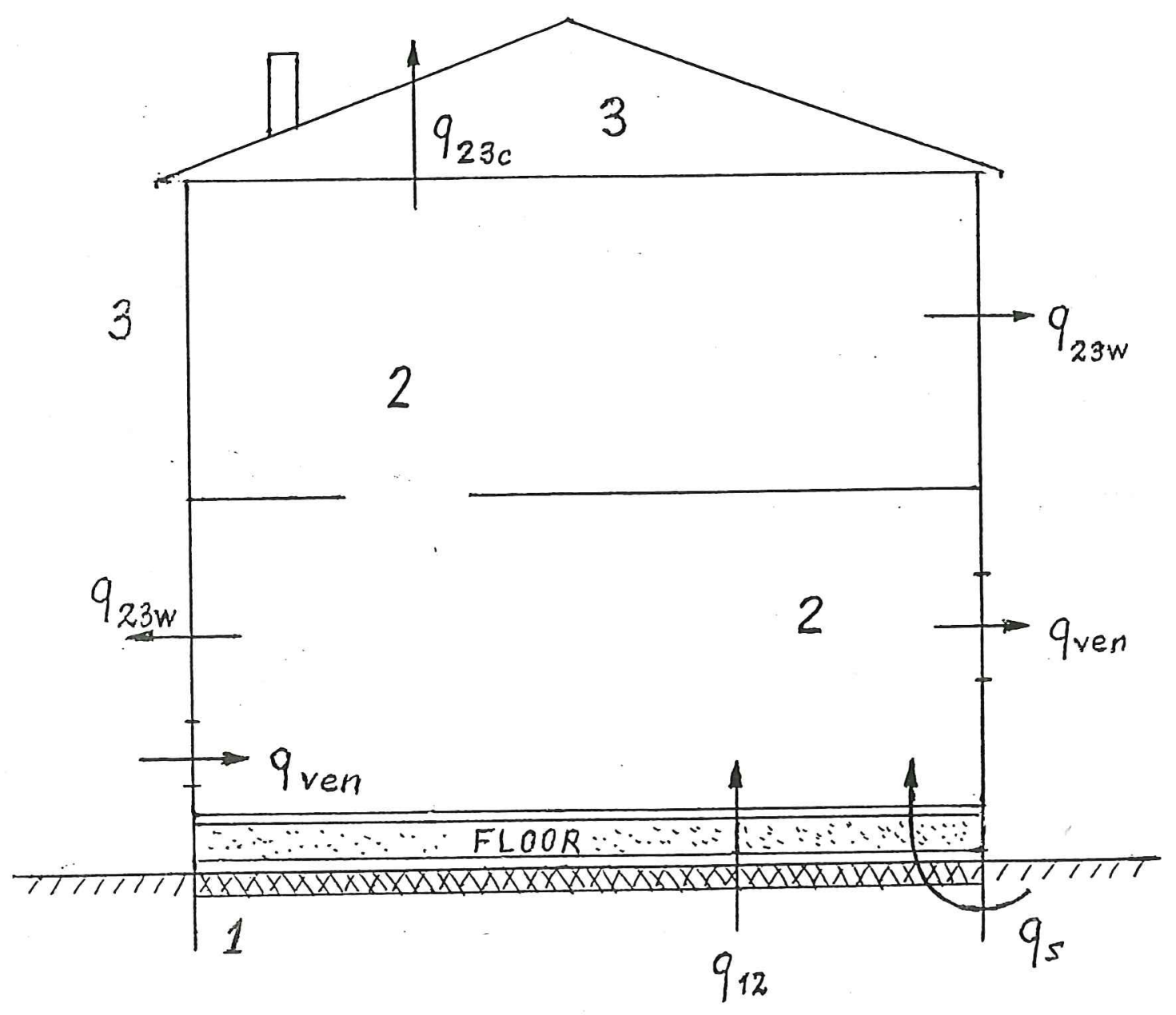

Fig. 1 


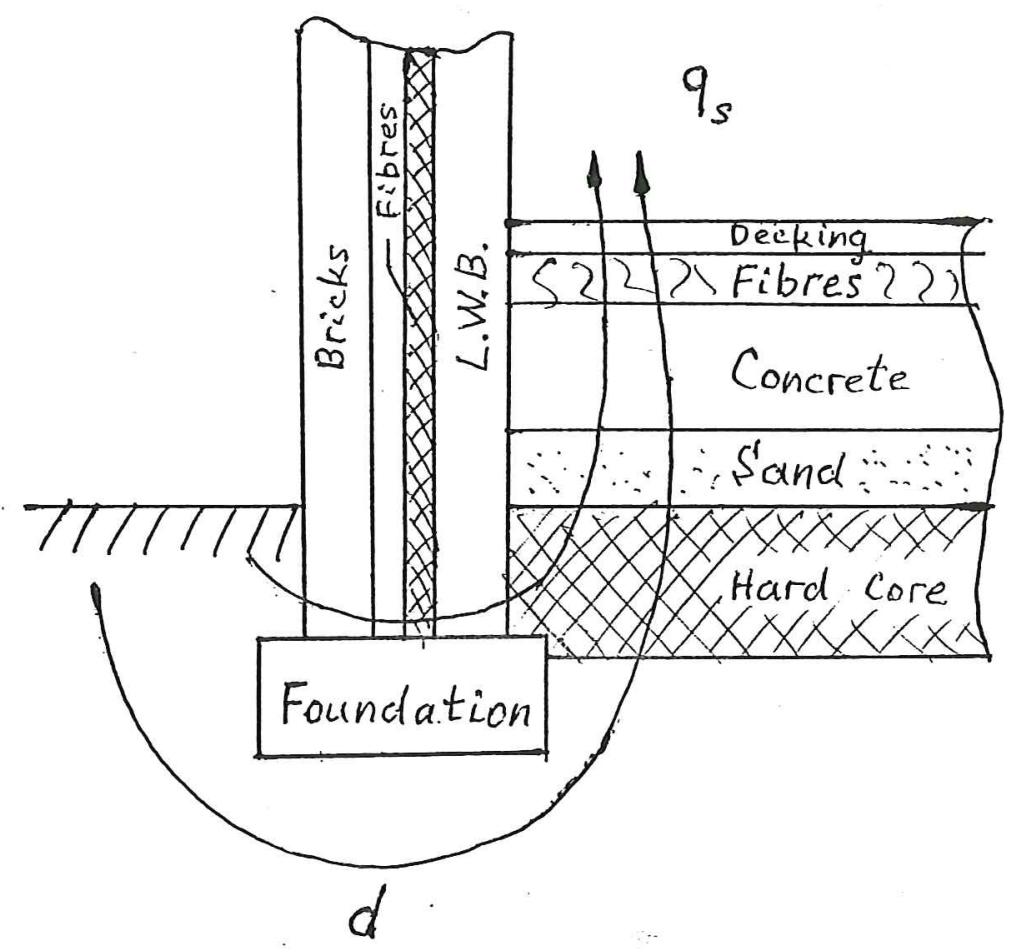

Fig. 2 


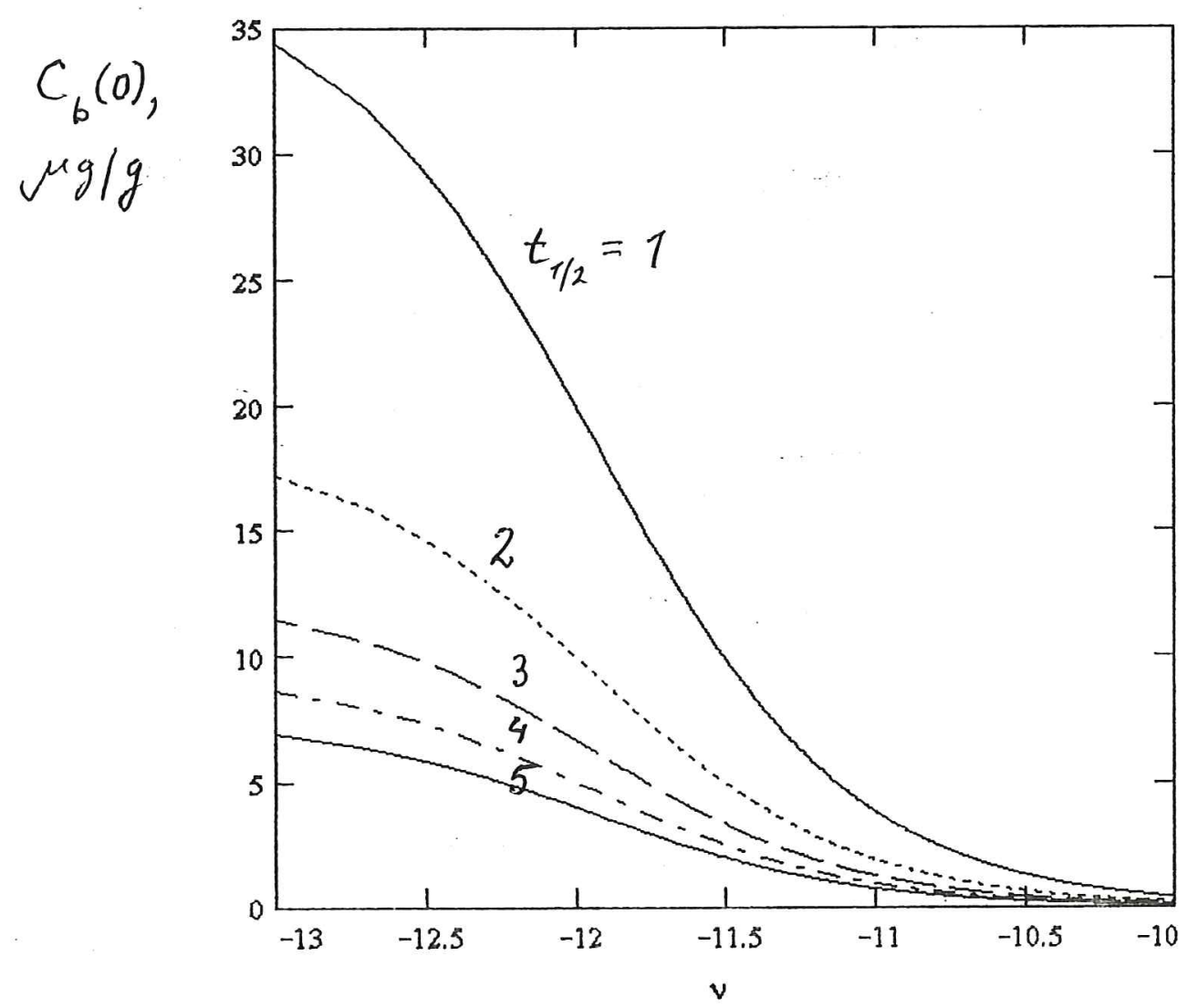

Fig. 3 


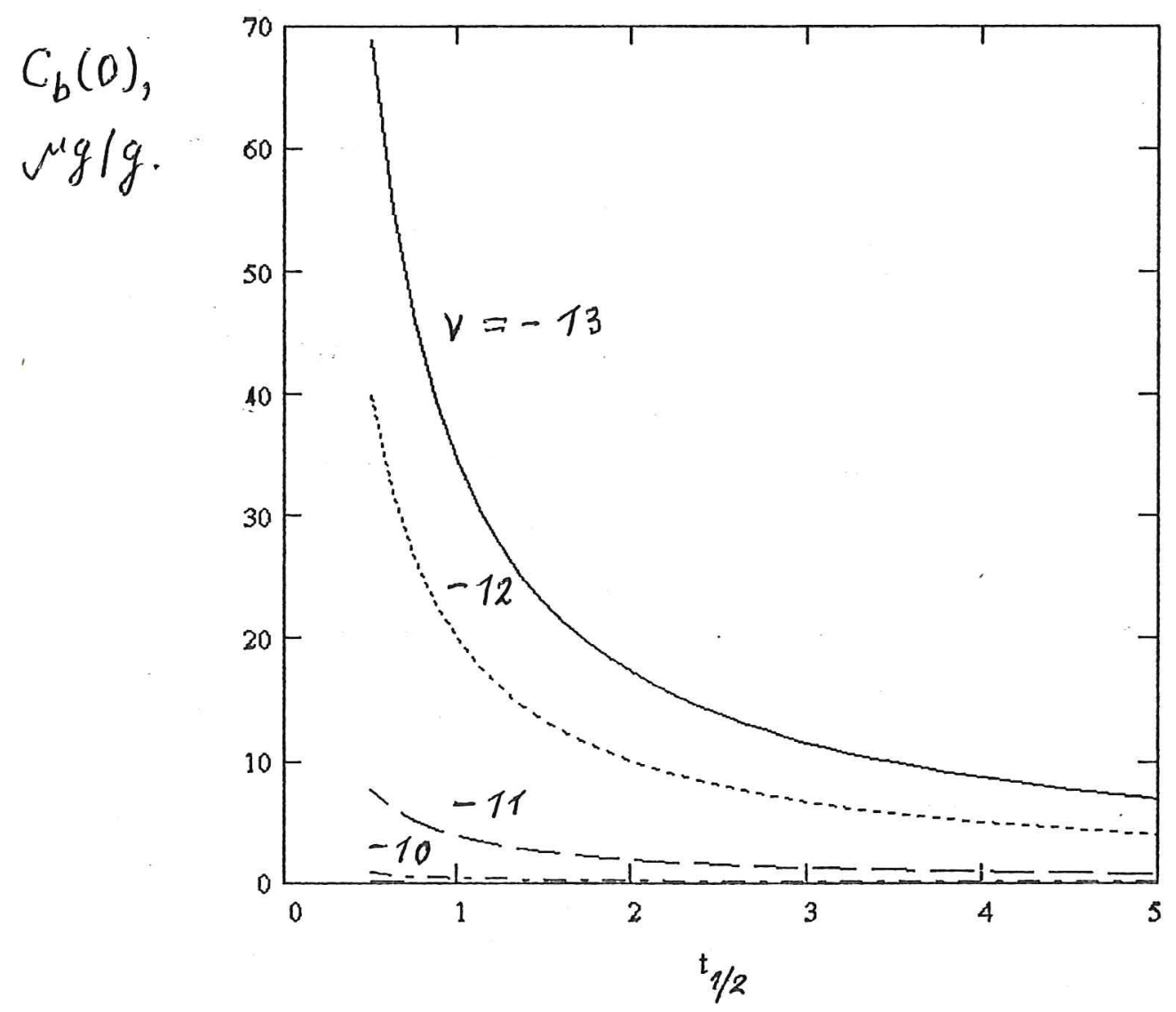

Fig. 4 


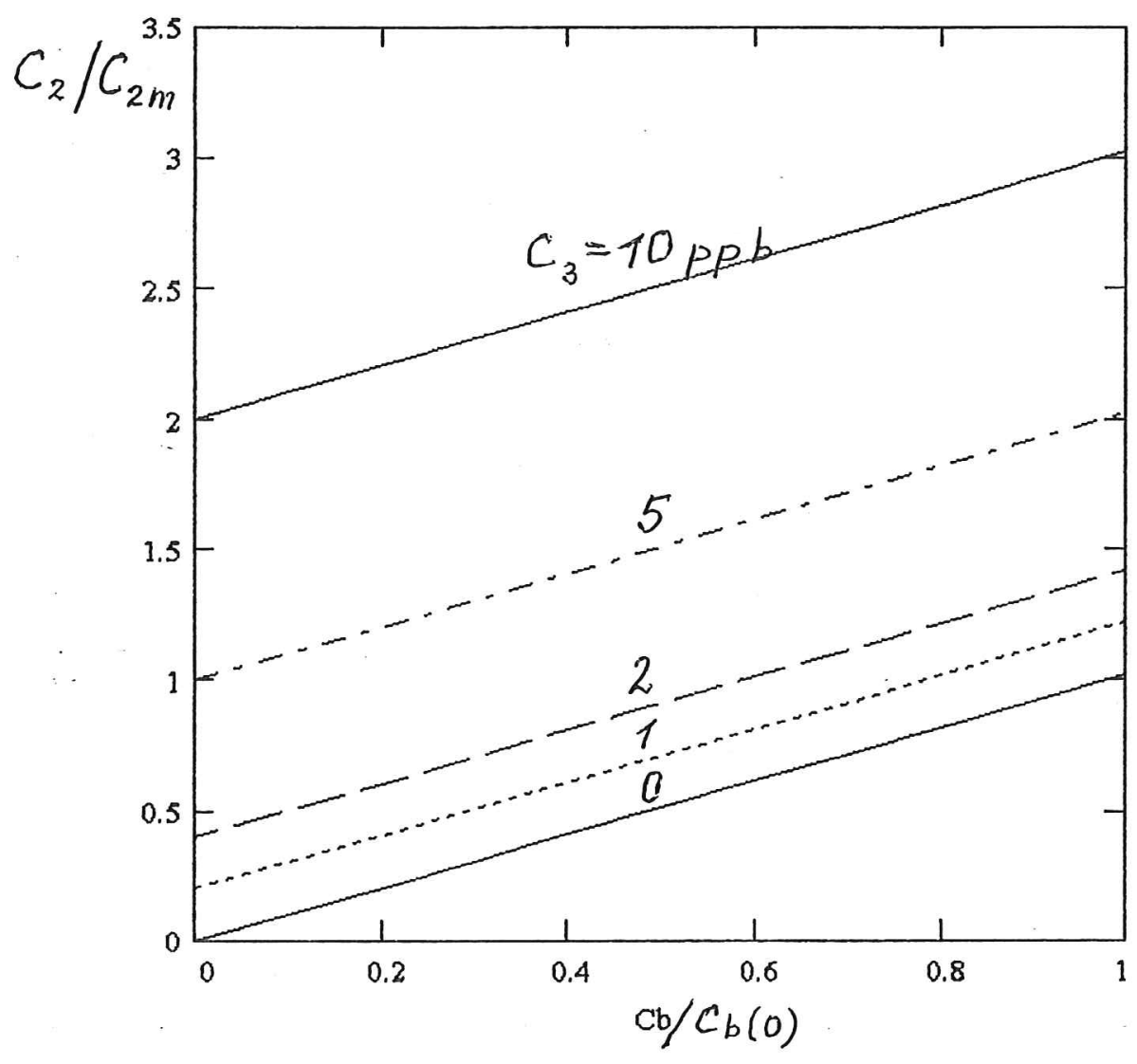

Fig. 5 